\title{
Implementation of a Communication Satellite Orbit Controller Design Using State Space Techniques
}

\author{
M. T. HLA ${ }^{*}$, Y. M. LAE ${ }^{2}$, S. L. KYAW ${ }^{3}$ AND M. N. ZAW \\ ${ }^{1}$ Department of Electronic Engineering, Mandalay Technological University, Patheingyi \\ Township, Republic of the Union of Myanmar \\ ${ }^{2,3}$ Department of Electronic Engineering, Mandalay Technological University, \\ Patheingyi Township, Republic of the Union of Myanmar \\ ${ }^{4}$ Technological University (Maubin), Maubin, Republic of the Union of Myanmar
}

\begin{abstract}
This research is of great importance for controlling the altitude of a satellite, especially one used for global communications in a geo-stationary orbit. The objective of this research was to advance a design based on the modelling of an orbit controller for a satellite orbiting into a circular orbit. This encompasses a good understanding of the system's dynamics. Once a satellite is launched into a desired orbit, it never remains in this ideal orbit. The external forces present in space cause perturbations to the ideal orbit. To bring the satellite back into the desired orbit, on-board thrusters provide the in-orbit propulsion. In this research, the altitude of the satellite was controlled by a thruster fashioned by the on-board thrusters installed in the radial and tangential directions. However, dictated by the controllable prerequisite, we achieved dynamic system stabilization with the aid of two thrusters as well as one thruster. Thus, the feedback dynamic control system responded to both the two-input and the single-input cases. The model developed was effectively a linearized, normalized and state-space model. The simulation of this model was based on the MATLAB environment. The design evolved accordingly was used to revise the effect of pole placement on the controlling parameters, such as settling time, peak time, overshoot, and damping ratio of the closed-loop system. This enabled us to make predictions on the stability requirements for several dynamic systems of the type considered. The design tool thus developed was applied to an actual current communication satellite design. The design results were evaluated and recommendations completed.
\end{abstract}

Key words: orbit controller design; state space analysis; MATLAB; communication satellite; advanced control techniques; simulation; feedback dynamic control system; stability requirements

Satellites offer the unique possibility of interconnecting users, regardless of their location or distance, and providing a complete spectrum of telecommunication services regardless of bandwidth requirements (Richharia \& Westbrook 2011). A satellite also needs a guidance system to make sure that it maintains the proper angle in relation to the earth. Communications satellites, weather forecasting satellites and remote-sensing satellites all have different subsystems. These spacecraft require major subsystems. They are the propulsion subsystem, thermal control subsystem, power supply subsystem, telemetry tracking and control subsystem, and altitude and orbit control subsystem. A satellite is launched into its approximate desired orbit by a large rocket, which may be carrying several satellites at the same time. Once a satellite is launched into a desired orbit, it never remains in this ideal

* Corresponding author (e-mail: kohlamyotun@gmail.com) 
orbit. This is because the external forces present in space cause perturbations to the ideal orbit. The satellite then uses its own main propulsion system to go into its final orbit, correcting for any unavoidable errors from the initial launch. Once a satellite has achieved its correct orbit, auxiliary rocket engines or thrusters are used to turn the satellite to the correct orientation in relation to the earth and the sun, and to perform many small station-keeping corrections for overcoming orbital distortions. There is a large range of satellite orbits, but not all of them are of use for satellite communications (Dorf \& Bishop 2011). The most commonly used orbit for fixed communications is the 24-hour geostationary orbit with an altitude of 35786 km (Maini \& Agrawal 2011). Modern satellite communication systems utilize geostationary satellites because they can provide significant advantages in achieving round-the-clock communications and tracking of the satellite. An altitude control system for a satellite vehicle within the earth's atmosphere is shown in Figure 1. The space satellite uses a control system to adjust its angular speed $\omega(t)$ and its angular position $\theta(t)$ for complete control of the satellite. This system requires two inputs in the radial and tangential directions to control the satellite. Thus, a state variable feedback controller is used for the altitude control system. In this research, the design of the controller utilizing state feedback is expressed. The basic principle of the feedback controller is the use of the pole placement technique that provides a unique solution.

\section{Principles of Orbiting Satellites}

The motion of natural and artificial satellites around the earth is governed by two forces. One of them is the centripetal force directed towards the centre of the earth due to the earth's gravitational force of attraction, while the other is the centrifugal force that acts outwards from the centre of the earth as shown in Figure 2. In the case of a satellite orbiting the earth, the satellite exerts a centrifugal force. In the absence of the earth's centripetal force, the satellite would have continued to move in a straight line at a constant speed after injection. The centripetal force directed at right angles to the satellite's velocity towards the centre of the earth transforms the straight line motion to a circular or elliptical one, depending upon the satellite's velocity. This is in accordance with Newton's third law of motion, which states that for every action there is an equal and opposite reaction (Maral 2009).

\section{Mathematical Model}

The planar motion of an orbiting satellite is considered in the inverse-square gravitational field of the earth when carrying out the mathematical modelling of the system. For simplicity's sake, the satellite is approximated to a particle of mass $M_{s}$. In the geostationary (circular) orbit, the gravitational force, which follows the universal law of gravity being equal to the required centripetal force, yields the description of the orbit. The orbit can be expressed in terms of the gravity at the orbit.

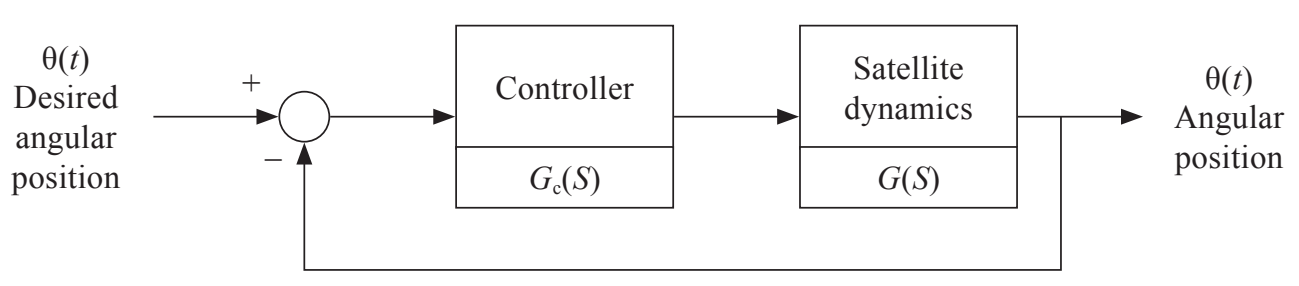

Figure 1. Block diagram of satellite altitude control. 


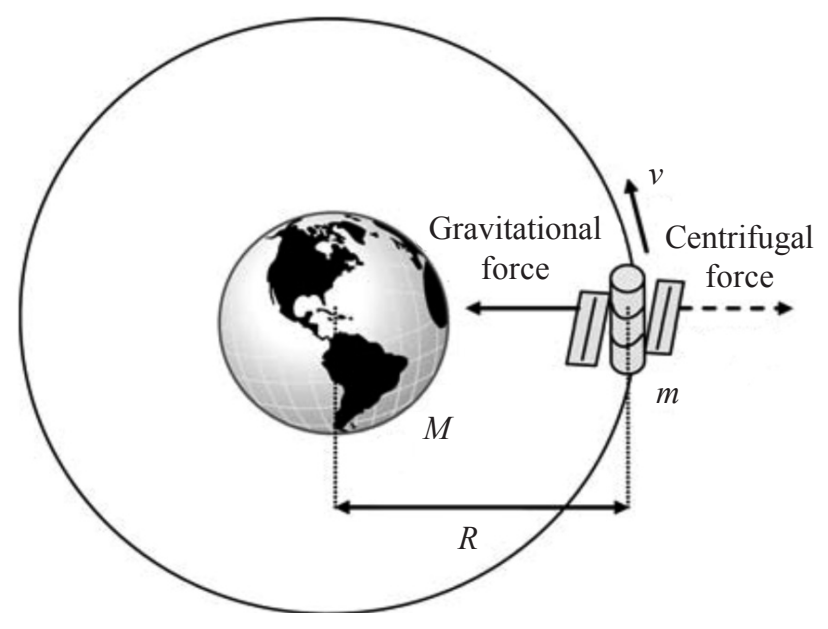

Figure.2. Two forces in orbiting earth.

The earth's varying gravitational field at varying altitudes may be written as:

$$
\begin{aligned}
g_{\text {orbit }} & =g_{\text {surface }}\left[\frac{R_{\text {earth }}}{r}\right]^{2} \\
g & =g\left(\frac{R_{\mathrm{e}}}{R_{e}+h}\right)^{2}
\end{aligned}
$$

where, $R_{e}=$ the radius of the earth, and

$$
h=\text { the altitude of the satellite from the }
$$
surface of the earth.

$$
r(t)=R_{e}+h
$$

where, $r(t)$ is the distance from the centre of the earth to the centre of the satellite.

Using Newton's third law of motion to substitute:

$$
F_{g}=M_{s} g\left(\frac{R_{\mathrm{e}}}{R_{e}+h}\right)^{2}
$$

we get:

$$
F_{g}=M_{s} g\left(\frac{R_{\mathrm{e}}}{r(t)}\right)^{2}
$$

This system involves a circular motion around a fixed centre, and thrusts are defined with respect to the tangential and radial directions as shown in Figure 3. The satellite motion is more conveniently described by its polar co-ordinates. In this situation, it is often more convenient to represent all variables in vector form with complex numbers.

The radial thrust vector is:

$$
F_{1}=F_{1} \mathrm{re}^{j \theta}
$$

The tangential thrust vector is:

$$
F_{2}=F_{2} \mathrm{re}^{j(\theta+90)}
$$

The gravitational force is:

$$
F_{g}=-M_{s} g\left(R^{2} / r^{2}\right) \mathrm{re}^{j \theta}
$$

The inertial force vector is

$$
F=M a=M_{s} \frac{d^{2} r}{d t^{2}}
$$

Newton's second law can be expressed mathematically as follows: where $\Sigma F$ is the vector sum of all the forces, $M_{s}$ is the 


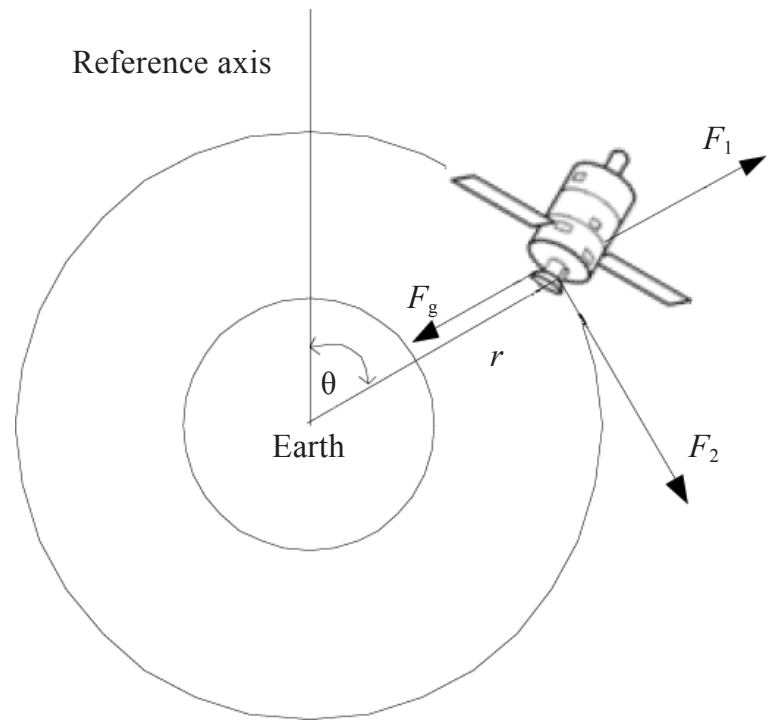

Figure 3. Circular movement of a communication satellite model (Richharia \& Westbrook 2011).

mass of the satellite, and is the vector acceleration of the mass measured relative to an interval reference form.

$$
\begin{gathered}
\sum F=M_{\mathrm{s}} \ddot{r} \\
F_{1}+F_{2}+F_{3}=M_{s} \frac{d^{2} r}{d t^{2}}=M_{s} \frac{d^{2}}{d t^{2}}\left[\mathrm{r}(\mathrm{t}) \mathrm{re}^{j \theta}\right]
\end{gathered}
$$

Therefore,

$$
\begin{aligned}
& F_{1} e^{\mathrm{j} \theta}+j F_{2} e^{\mathrm{j} \theta}-M_{\mathrm{s}} g\left(\frac{R^{2}}{r^{2}}\right) e^{\mathrm{j} \theta}=M_{s}\left[\ddot{r} e^{\mathrm{j} \theta}-r \dot{\theta}^{2} e^{\mathrm{j} \theta}+j\left(r \dot{\theta} e^{\mathrm{j} \theta}+2 \dot{\theta} \dot{r} e^{\mathrm{j} \theta}\right)\right] \\
& {\left[F_{1}-M_{\mathrm{s}} g\left(\frac{R^{2}}{r^{2}}\right) e^{\mathrm{j} \theta}\right]+j F_{2} e^{\mathrm{j} \theta}=\left[\left(M_{s} \ddot{r}-M_{s} r \dot{\theta}^{2}\right) e^{\mathrm{j} \theta}+j\left(M_{s} \dot{r} \ddot{\theta}+2 M_{s} \dot{\theta} \dot{r}\right) e^{\mathrm{j} \theta}\right]}
\end{aligned}
$$

By cancelling the common factor and equating the real part and the imaginary part, respectively, of Equation 13, two second-order differential equations are produced:

$$
\begin{aligned}
& F_{1}=M_{\mathrm{s}} \ddot{r}-M_{s} r \dot{\theta}^{2}+M_{\mathrm{s}} g\left(\frac{R^{2}}{r^{2}}\right) \\
& F_{2}=M_{s} \dot{r} \ddot{\theta}+2 M_{s} \dot{\theta} \dot{r}\left(\frac{R^{2}}{r^{2}}\right)
\end{aligned}
$$


Hence, Equation 14 and Equation 15 can be rewritten as follows:

$$
\begin{aligned}
& \frac{F_{1}}{M_{\mathrm{s}} g}=\frac{\ddot{r}}{g}-\frac{r \dot{\theta}^{2}}{g}+\frac{R^{2}}{r^{2}} \\
& \frac{F_{2}}{M_{\mathrm{s}} g}=\frac{r \ddot{\theta}}{g}+\frac{2 \dot{\theta} \dot{r}}{g}
\end{aligned}
$$

To simplify the numerical problem, the time, distance and force variables are normalized into dimensionless quantities.

$$
\text { Let } \begin{aligned}
\tau & =\frac{\tau}{\left(\frac{R}{g}\right)^{1 / 2}} ; \rho=\frac{\mathrm{r}}{R} ; \\
u_{1} & =\frac{F_{1}}{M_{\mathrm{s}} g} ; u_{1}=\frac{F_{2}}{M_{\mathrm{s}} g}
\end{aligned}
$$

where, $\tau=$ settling time, $\rho=$ constant, $u_{1}=$ radial thruster force, $u_{2}=$ tangential thruster force; then:

$$
u_{1}=\frac{\ddot{r}}{g}-\frac{r \dot{\theta}^{2}}{g}+\frac{R^{2}}{r^{2}}
$$

Further,

$$
u_{2}=\frac{r \ddot{\theta}}{g}+\frac{2 \dot{\theta} \dot{r}}{g}
$$

When we wish to change from the function $(t)$ to the function $(\tau)$, we can use the following relationship:

$$
r=\frac{d r(t)}{d t}=\frac{d}{d \tau}\left(\frac{g}{R}\right)^{1 / 2} r(\tau)
$$

This Equation 21 may be rewritten as:

$$
\dot{r}(t)=\dot{r}(t) \times\left(\frac{g}{R}\right)^{1 / 2}
$$

$$
\begin{aligned}
\ddot{r}(\mathrm{t})=r^{\prime \prime}(\mathrm{t}) \times\left(\frac{g}{R}\right)^{1 / 2} \times\left(\frac{g}{R}\right)^{1 / 2} & \\
= & r^{\prime \prime}(\mathrm{t}) \times\left(\frac{g}{R}\right) \\
\text { Similarly, } \dot{\theta}(\mathrm{t}) & =\dot{\theta}(\tau) \times\left(\frac{g}{R}\right)^{1 / 2} \\
\dot{\theta}^{2}(t) & =\dot{\theta}^{2}(\tau)\left(\frac{g}{R}\right) \\
\dot{\theta}^{2}(t) & =\dot{\theta}^{2}(\tau)\left(\frac{g}{R}\right)
\end{aligned}
$$

By substituting Equation 22 and Equation 23 into Equation 26,

$$
u_{1}=\rho^{\prime \prime}-\rho \dot{\theta}^{2}(\tau)+\left(\frac{1}{\rho^{2}}\right)
$$

Then,

$$
u_{2}=2 \dot{\rho}(\tau) \dot{\theta}(\tau)+\rho \theta^{\prime \prime}(\tau)
$$

The system of the first-order ordinary differential equations is considered. By defining the four-state variables as below, this will result in a set of differential equations.

Let $x_{1}=\rho ; x_{2}=\theta ; \dot{x}_{1}=\dot{\rho}=\dot{\mathrm{x}}_{1}=\dot{\rho}=x_{3}$;

$\dot{x}_{2}=\dot{\theta}=x_{4} ; \dot{x}_{3}=\ddot{\rho} ; \dot{x}_{4}=\ddot{\theta}$

$$
\text { Hence, } \rho^{\prime \prime}=u_{1}+\rho \dot{\theta}^{2}(\tau)-\frac{1}{\rho^{2}}
$$

This Equation 27 may be rewritten as:

$$
\dot{x}_{3}=x_{1} x_{4}^{2}-\frac{1}{x_{4}^{2}}+\mathrm{u}_{1}
$$

Hence, from Equation 28

$$
\dot{x}_{4}=-\frac{2 x_{3} x_{4}}{x_{1}}+\frac{1}{x_{1}} \mathrm{u}_{2}
$$


Thus, a non-linear state model is obtained as given below:

$$
\begin{aligned}
& x_{1}^{\prime}=x_{3}, \dot{x}_{2}=x_{4} \\
& x_{3}^{\prime}=x_{1} x_{4}^{2}-\frac{1}{x_{4}^{2}}+\mathrm{u}_{1} \\
& x_{4}^{\prime}=-\frac{2 x_{3} x_{4}}{x_{1}}+\frac{1}{x_{1}} \mathrm{u}_{2}
\end{aligned}
$$

\section{Linearized System Model}

A mathematical model of a geostationary communications satellite in the earth's equatorial plane was derived. It was necessary the system to have a linearized state-space model with the geostationary orbit. The non-linear state model equation can be solved by using the Jacobian matrix to obtain the linearized equation (Dorf \& Bishop 2011).

The Jacobian matrix equation of:

$$
f_{1}\left(x_{1}, x_{2}, x_{3}, x_{4}\right)=\left[f_{1}\left(x_{1}, x_{2}, x_{3}, x_{4}\right), f_{1}\left(x_{1}, x_{2}, x_{3}, x_{4}\right), f_{1}\left(x_{1}, x_{2}, x_{3}, x_{4}\right), f_{1}\left(x_{1}, x_{2}, x_{3}, x_{4}\right)\right]^{T}
$$

is evaluated at $\left(x_{1}, x_{2}, x_{3}, x_{4}\right)^{T}=(0,0,0,0)^{T}$

$$
J(0)=A=\left[\begin{array}{cccc}
0 & 0 & 1 & 0 \\
0 & 0 & 0 & 1 \\
x_{4}^{2}+\frac{2}{x_{1}^{3}} & 0 & 0 & 2 x_{1} x_{4} \\
0 & 0 \frac{-2 x_{4}}{x_{1}} & 0
\end{array}\right]
$$

Thus, the linearized and normalized equations of motion in the geostationary orbit are given by:

$$
\left[\begin{array}{c}
\delta x_{1}^{\prime} \\
\delta x_{2}^{\prime} \\
\delta x_{3}^{\prime} \\
\delta x_{4}^{\prime}
\end{array}\right]=\left[\begin{array}{cccc}
0 & 0 & 1 & 0 \\
0 & 0 & 0 & 1 \\
x_{4}^{2}+\frac{2}{x_{1}^{3}} & 0 & 0 & 2 x_{1} x_{4} \\
0 & 0 & \frac{-2 x_{4}}{x_{1}} & 0
\end{array}\right]\left[\begin{array}{l}
\delta x_{1} \\
\delta x_{2} \\
\delta x_{3} \\
\delta x_{4}
\end{array}\right]+\left[\begin{array}{cc}
0 & 0 \\
0 & 0 \\
1 & 0 \\
0 & \frac{1}{x_{1}}
\end{array}\right]\left[\begin{array}{l}
u_{1} \\
u_{2}
\end{array}\right]
$$

Therefore, according to Equation 36, we can express-the following:

$$
x_{1}=\rho=\frac{r}{R_{e}}=\frac{R_{e}+h_{0}}{R_{e}}
$$




$$
\begin{aligned}
& x_{2}=\theta \\
& x_{3}=\dot{\rho}=0 \\
& x_{4}=\dot{\theta}=\omega_{0}
\end{aligned}
$$

According to the above statements, the other type of state model equation is:

$$
\left[\begin{array}{l}
\delta x_{1}^{\prime} \\
\delta x_{2}^{\prime} \\
\delta x_{3}^{\prime} \\
\delta x_{4}^{\prime}
\end{array}\right]=\left[\begin{array}{cccc}
0 & 0 & 1 & 0 \\
0 & 0 & 0 & 1 \\
\omega^{2}+\frac{2}{\rho^{3}} & 0 & 0 & 2 r \omega \\
0 & 0 & \frac{-2 \omega}{r} & 0
\end{array}\right]\left[\begin{array}{l}
\delta x_{1} \\
\delta x_{2} \\
\delta x_{3} \\
\delta x_{4}
\end{array}\right]+\left[\begin{array}{cc}
0 & 0 \\
0 & 0 \\
1 & 0 \\
0 & \frac{1}{r}
\end{array}\right]\left[\begin{array}{l}
u_{1} \\
u_{2}
\end{array}\right]
$$

\section{Steady State Components of Two-thrust Vectors}

For geometric consideration, a satellite in a stable circular orbit around the earth can be expressed as $F_{\text {gravity }}=F_{\text {centripetal. }}$. From this relationship, the period of the orbit and the orbital velocity or angular velocity of the satellite can be calculated. The most commonly used satellite orbit for fixed communications is the 24-hour geostationary (circular) orbit with an altitude of $35786 \mathrm{~km}$. The satellite period of rotation around the earth is $86157.8836 \mathrm{~s}$, or $23 \mathrm{~h}, 55 \mathrm{~min}$ and $77 \mathrm{~s}$, and the angular velocity is 15.04 degrees per hour. Once the simulation is generated, a generalized case of the satellite orbiting into a circular orbit at any altitude can be created. The altitude choice and the technique for the synchronous satellite are provided by applying the illustration of coverage area and geometric satellite orbits. Using empirical values $\left(R_{e}=6378 \mathrm{~km}, \mathrm{~h}=35786 \mathrm{~km}\right.$, $\mathrm{r}=42164 \mathrm{~km}, \mathrm{~g}=9.8087 \mathrm{~m} / \mathrm{s}^{2}$ ), the apex angle $2 \alpha$ equals 17.4 degrees, while the planar angle beamwidth. Now suppose the satellite is to be maintained in a circular geostationary orbit of the earth. The steady state is maintained only by the gravitational force, so that the steady state components of two-thrust vectors are zero. To minimize energy consumption, thrusts are only applied to take transient corrective action to eliminate error.

$u_{1}=0, \quad u_{2}=0$

$x_{3}=0, \quad x_{4}$ (angular speed) is a constant.

Therefore, $x_{4}$ and all the remaining variables can be determined.

$$
\omega=15.04 \mathrm{deg} / \mathrm{h}=\frac{\pi}{43200} \operatorname{rad} \text { per sec. }
$$

This corresponds to a normalized angular speed of:

$$
x_{4}=(R / g)^{1 / 2} \omega=0.05866
$$

The satellite maintains a nominal orbit as long as there are no disturbances. If the perturbations are sufficiently small $(\mathrm{E}<<1)$, they may be described by a linearized system. Here, the non-linear 
model and linearized model equations are both of the fourth order, and apply to situations in which not only the angular speed $x_{4}=(t)$ or $\omega(t)$ needs to be regulated, but also the angular position $\theta(\mathrm{t})$ must be accurately controlled for complete control of the satellite. So far, this all implies that the system requires two inputs in the radial and tangential directions to control the satellite. However, the system will be further checked to find out the different possibilities for controllability of the system.

In the state space equation, $R_{e}=6378 \mathrm{~km}$ and $h_{0}=35786 \mathrm{~km}$,

$$
\begin{aligned}
& x_{1}=\frac{r}{R}=\frac{R_{e}+h_{0}}{R_{e}}=\frac{6378 \mathrm{~km}+35786 \mathrm{~km}}{6378 \mathrm{~km}}=6.6108 \\
& \omega=15.04 \mathrm{deg} / \mathrm{h}=\frac{\pi}{43200} \text { rad per sec }=7.2915 \times 10^{-5} \mathrm{rad} / \mathrm{s}
\end{aligned}
$$

Normalized angular speed or $\omega=\left(\frac{R}{g}\right)^{1 / 2} \times 7.2915 \times 10^{-5}=0.05873$

$$
A=\left[\begin{array}{cccc}
0 & 0 & 1 & 0 \\
0 & 0 & 0 & 1 \\
x_{4}^{2}+\frac{2}{x_{1}^{3}} & 0 & 0 & 2 x_{1} x_{4} \\
0 & 0 & \frac{-2 x_{4}}{x_{1}} & 0
\end{array}\right]+\left[\begin{array}{cccc}
0 & 0 & 1 & 0 \\
0 & 0 & 0 & 1 \\
0.01036 & 0 & 0 & 0.7753 \\
0 & 0 & -0.1774 & 0
\end{array}\right]
$$

The linearized and normalized equations of motion in the geostationary orbit are given by:

$$
\left[\begin{array}{l}
\delta x_{1}^{\prime} \\
\delta x_{2}^{\prime} \\
\delta x_{3}^{\prime} \\
\delta x_{4}^{\prime}
\end{array}\right]=\left[\begin{array}{cccc}
0 & 0 & 1 & 0 \\
0 & 0 & 0 & 1 \\
0.01036 & 0 & 0 & 0.7753 \\
0 & 0 & -0.1774 & 0
\end{array}\right]\left[\begin{array}{c}
\delta x_{1} \\
\delta x_{2} \\
\delta x_{3} \\
\delta x_{4}
\end{array}\right]+\left[\begin{array}{cc}
0 & 0 \\
0 & 0 \\
1 & 0 \\
0 & 0.1511
\end{array}\right]\left[\begin{array}{l}
u_{1} \\
u_{2}
\end{array}\right]
$$

\section{Position Control with Two-thrust State Feedback}

In the first case, the satellite is being controlled by radial and tangential thrusts. Then the satellite can be regulated by its angular speed $x_{4}(t)$ or $\omega(t)$. The angular position $\theta(t)$ must also be controlled, and it is assumed that all the four state variables are available. The multi-input state variable systems require feedback control. This feedback system can de-couple into two single-input to overcome the two-input problem as shown in Figure 4. Here, the state variables $\delta x_{1}$ and $\delta x_{3}$ are responsible for changing the radial position of the satellite, while the state variables $\delta x_{2}$ and $\delta x_{4}$ cause a change to the angular position of the satellite. 


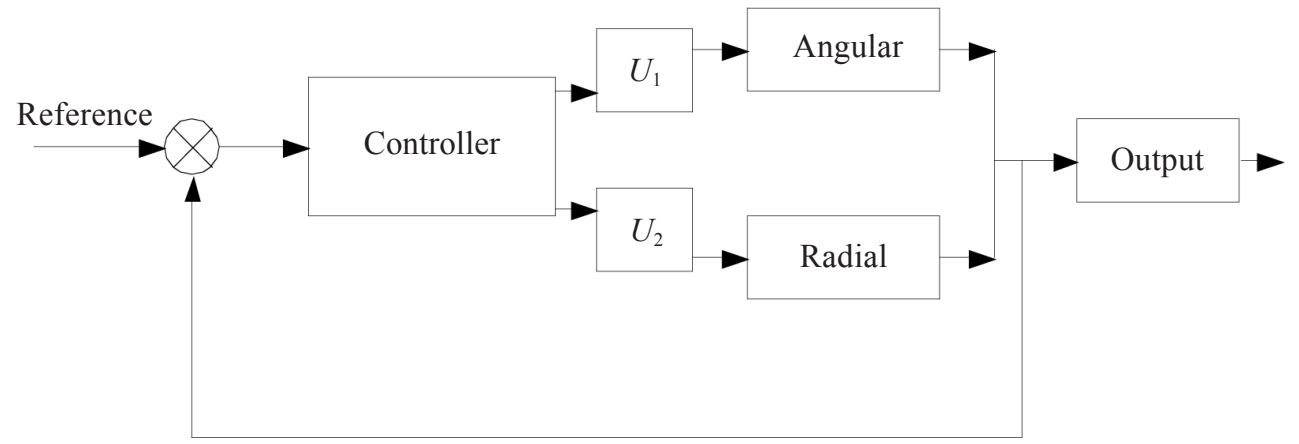

Figure 4. Decoupled two single-input feedback (Malik, Zaidi \& Khushnood 2001).

While carrying out the de-coupling, the third row of matrix A from the linearized model equations can be arbitrarily changed with the state feedback applied to $u_{\mathrm{r}}$ ( or $\left.u_{1}\right)$.

$$
\left[\begin{array}{l}
\delta x_{1}^{\prime} \\
\delta x_{2}^{\prime} \\
\delta x_{3}^{\prime} \\
\delta x_{4}^{\prime}
\end{array}\right]=\left[\begin{array}{cccc}
0 & 0 & 1 & 0 \\
0 & 0 & 0 & 1 \\
0.01036 & 0 & 0 & 0.7753 \\
0 & 0 & -0.1774 & 0
\end{array}\right]\left[\begin{array}{c}
\delta x_{1} \\
\delta x_{2} \\
\delta x_{3} \\
\delta x_{4}
\end{array}\right]+\left[\begin{array}{l}
0 \\
0 \\
1 \\
0
\end{array}\right]\left[u_{1}\right]
$$

Then, let $u_{1 \mathrm{a}}=-0.01036 \delta x_{1}-0.7753 \delta x_{4}$, putting it in the third row of the linearized model equations.

So, a single-input second-order system can be written as:

$$
\begin{aligned}
& \delta x_{1}^{\prime}=\delta x_{3} \\
& \delta x_{3}^{\prime}=0.01036 \delta x_{1}+0.7753 \delta x_{4}-0.01036 \delta x_{1}-0.7753 \delta x_{4} \\
& \delta x_{3}^{\prime}=u_{1 \mathrm{~b}}
\end{aligned}
$$

thus, producing a single-input second-order system as in Equation 48.

$$
\delta x_{1}^{\prime}=\delta x_{3}, \delta x_{3}^{\prime}=u_{1 \mathrm{~b}}
$$

Control of the radial variables is separated from the state model equation. Thus, the above second-order model equation describes the control of the angular variables. The response of the double-integrator system equation can easily be controlled to provide a desired characteristic equation.

Applying the inverse Laplace transformation on both sides of the equation:

$$
y^{\prime \prime}+2 \xi \omega_{\mathrm{n}} y^{\prime}+\omega_{\mathrm{n}}^{2} y=0
$$


Let $\delta x_{1}=y, \delta x_{1}^{\prime}=y^{\prime}=\delta x_{3}, \delta x_{3}^{\prime}=y^{\prime \prime}=-2 \xi \omega_{n} y^{\prime}-\omega_{n}^{2} y, \delta x_{3}^{\prime}=u_{1 \mathrm{~b}}=-2 \xi \omega_{n} y^{\prime}-\omega_{n}^{2} y$

and by selecting $\xi=0.707$ (say) to meet the overshoot requirement, the settling time of 12 hours can expressed by:

$$
\begin{aligned}
& \tau_{s}=\frac{4}{\xi \omega_{n}}=53.5731 \\
& \omega_{n}=0.1056
\end{aligned}
$$

The settling time specification is then easily translated into $\omega_{n}=0.1056$. The second-order model equation becomes:

$$
\begin{aligned}
& \delta x_{1}^{\prime}=\delta x_{3} \\
& \delta x_{3}^{\prime}=-0.0115 \delta x_{1}-0.1493 \delta x_{3}
\end{aligned}
$$

Hence, the overall state feed law for the radial thrust is:

$$
u_{1}=-0.0215 \delta x_{1}-0.1493 \delta x_{3}-0.7753 \delta x_{4}
$$

In exactly the same manner, the angular dynamics described in the second and the fourth rows of the matrix A of the state-model equation can be de-coupled with state feedback applied to the tangential thrust $\mathrm{u}_{\mathrm{t}}\left(\right.$ or $\left.u_{2}\right)$.

$$
\left[\begin{array}{l}
\delta x_{1}^{\prime} \\
\delta x_{2}^{\prime} \\
\delta x_{3}^{\prime} \\
\delta x_{4}^{\prime}
\end{array}\right]=\left[\begin{array}{cccc}
0 & 0 & 1 & 0 \\
0 & 0 & 0 & 1 \\
0.01036 & 0 & 0 & 0.7753 \\
0 & 0 & -0.1774 & 0
\end{array}\right]\left[\begin{array}{c}
\delta x_{1} \\
\delta x_{2} \\
\delta x_{3} \\
\delta x_{4}
\end{array}\right]+\left[\begin{array}{c}
0 \\
0 \\
0 \\
0.1511
\end{array}\right]\left[u_{2}\right]
$$

Let $u_{2 \mathrm{a}}=\frac{0.1774}{0.1511} \delta x_{3}=0.1774 \delta x_{3}$, putting it in the third row of the linearized model equations.

So, a single-input second-order system can be written as:

$$
\delta x_{1}^{\prime}=\delta x_{3}^{\prime}, \delta x_{4}^{\prime}=0.1511 u_{2 \mathrm{~b}}
$$

thus, producing a single-input second-order system as in Equation 55

$$
\delta x_{1}^{\prime}=\delta x_{3}^{\prime} ; \delta x_{4}^{\prime}=0.1511 u_{2 \mathrm{~b}}
$$

Control of the tangential variables is separated from the state model equation. Thus, the above second-order model equation describes the control of the angular variables. The response of the double-integrator system equation can easily be controlled to provide a desired characteristic equation. 
Similarly, the two-input state feedback equations are:

$$
\begin{aligned}
& u_{1}=0.0215 \delta x_{1}-0.1493 \delta x_{3}-0.7753 \delta x_{4} \\
& u_{1}=0.07379 \delta x_{1}-0.1174 \delta x_{3}-0.9881 \delta x_{4}
\end{aligned}
$$

Therefore, the two-input state feedback control takes the form as follows:

$$
u=\left[\begin{array}{cccc}
-0.02151 & 0 & -0.1493 & -0.7753 \\
0 & -0.07379 & 0.1174 & -0.9881
\end{array}\right]\left[\begin{array}{l}
\delta x_{1}^{\prime} \\
\delta x_{2}^{\prime} \\
\delta x_{3}^{\prime} \\
\delta x_{4}^{\prime}
\end{array}\right]
$$

In this way, the satellite altitude can be controlled by the use of two thrusts in the radial and tangential directions which help in controlling both the angular speed $x_{4}(t)$ and $\omega(t)$, and the angular position $\theta(t)$ of the satellite with the application of the control parameters.

\section{State Feedback Control of Angular Rate with the Tangential Thrust}

After checking the controllability of the model equation, it was revealed that the satellite could also be controlled by only a single radial thrust by controlling the angular rate $\omega(t)$ of the satellite but with a constant non-zero offset error in the angular position $\theta(t)$. Moreover, this controlling technique can be employed in a situation where the radial thruster has failed and only the tangential thrust is applied to the MATLAB's ctrb function. This reduced model equation with the elimination of the radial thrust $u_{1}$ of the third order is shown in Equation 40 . The ' $\mathrm{C}$ ' matrix is essentially the required parameters that are of value from the input values.

$$
\begin{aligned}
& {\left[\begin{array}{l}
\delta x_{1}^{\prime} \\
\delta x_{2}^{\prime} \\
\delta x_{3}^{\prime}
\end{array}\right]=\left[\begin{array}{ccc}
0 & 1 & 0 \\
0.01036 & 0 & 0.7753 \\
0 & -0.01774 & 0
\end{array}\right]\left[\begin{array}{c}
0 \\
0 \\
0.1512
\end{array}\right]\left[u_{2}\right]} \\
& y=\mathrm{C} x+\mathrm{D} u, y=\left[\begin{array}{llll}
1 & 0 & 0 & 0
\end{array}\right], D=0
\end{aligned}
$$

\section{Pole Placement Using State Feedback}

This is a single-input system problem, which can be easily solved by pole placement that is able to provide a unique solution. In this situation, the three state variables are available for providing feedback. Energy efficiency consideration is one of the important factors in an aerospace system. Normally, pole placement is achieved by moving poles at least five times to the left of the s-plane compared with the dominant ones, thus making the analysis and design easier. This usually requires the use of a large control input, which is not feasible in an aerospace system. The input energy requirement can often be reduced if all the closed-loop poles are placed at about the same distance from the origin. Thus, the three poles are placed at an equal distance from the origin. 


$$
s_{1}=\omega_{n} e^{\mathrm{j} 120}, s_{2}=\omega_{n} e^{-\mathrm{j} 120}, s_{3}=\omega_{n} e^{\mathrm{j} 180}=\omega_{n},(\cos 180+j \sin 180)=-\omega_{n}
$$

For such three-pole systems, the third pole at $s_{3}=-\omega_{n}$ usually has an effect of lowering the overshoot and reducing the settling time. Thus, a conservative estimate of transient performance can be obtained by ignoring $s_{3}$ and considering only the effects of $s_{1}$ and $s_{2}$. For this system, the damping ratio chosen is $\xi=\cos 60=0.5$, which corresponds to $17 \%$ overshoot. This implies that the system requires a $2 \%$ settling time of 12 h, i.e. 53.53731 seconds as shown in Equation 50 in the previous discussion can be expressed.

$$
\tau_{s}=\frac{4}{\xi \omega_{n}}=53.5731, \omega_{n}=0.1495
$$

In this way, the state feedback gain for the third-order state model can be determined when all the closed-loop poles are placed at an equal distance from the origin, and correspond to a damping ratio of $\xi=0.5$ and $\omega_{n}=0.1495$. The desired characteristic equation is then $s^{3}+0.22395 s^{2}+0.03343$ $5 s+0.00166=0$.

For the third-order system with a close-loop transfer function, the equation is:

$$
\mathrm{T}(s)=\frac{1}{\left(s+\xi \omega_{n}\right)\left(s^{2}+2 \xi \omega_{n} s+\omega_{\mathrm{n}}^{2}\right)}
$$

The three-state variable feedback shown in $\dot{x}=A x+B u$ are selected.

$$
\left[\begin{array}{l}
\delta x_{1}^{\prime} \\
\delta x_{2}^{\prime} \\
\delta x_{3}^{\prime}
\end{array}\right]=\left[\begin{array}{ccc}
0 & 1 & 0 \\
0.01036 & 0 & 0.7753 \\
0 & -0.01774 & 0
\end{array}\right]\left[\begin{array}{c}
0 \\
0 \\
0.1512
\end{array}\right]\left[u_{2}\right]
$$

If the state variable matrix $K$ is $K=\left[k_{1} k_{2} k_{3}\right]$, and the feedback of the state variables is $u=-K x$, then $\dot{\mathrm{x}}=A x-B K x=(A-B K x)$.

The state feedback matrix is:

$$
\begin{aligned}
& \dot{x}=\left[\begin{array}{ccc}
0 & 1 & 0 \\
0.01036 & 0 & 0.7754 \\
0 & -0.01774 & 0
\end{array}\right]-\left[\begin{array}{c}
0 \\
0 \\
0.1511
\end{array}\right]\left[\begin{array}{lll}
k_{1} & k_{2} & k_{3}
\end{array}\right] \\
& \operatorname{det}[A-B K]=s^{2}+0.1511 s^{2}+(0.00345+0.1174) s-0.0016 k_{3}+0.1175 k_{1}
\end{aligned}
$$

when comparing with the values of $k, k_{1}=0.0343, k_{2}=0.2553$, and $k_{3}=1.48$. 
This shows that the pole placement requires $k_{1}=0.0343, k_{2}=0.2553, k_{3}=1.48$. Hence, the state feedback gain for the system with the desired pole placement is:

$$
K=\left(\begin{array}{lll}
0.0343 \quad 0.2553 \quad 1.48
\end{array}\right)
$$

After computing the state feedback gain vector, the close-loop transfer function is considered for steady-state tracking. The open-loop state equation is specified by the following coefficient matrices although they are in controller form.

$$
A=\left[\begin{array}{ccc}
0 & 1 & 0 \\
0.01036 & 0 & 0.7753 \\
0 & -0.01774 & 0
\end{array}\right], B=\left[\begin{array}{c}
0 \\
0 \\
0.1512
\end{array}\right], C=\left[\begin{array}{lll}
1 & 0 & 0
\end{array}\right]
$$

The open-loop transfer function is, by inspection,

$$
H(s)=\frac{0.1171 s}{s^{3}+0.00339 s}
$$

The state feedback gain was computed to be $K=\left[\begin{array}{lll}0.0343 & 0.2553 & 1.48\end{array}\right]$

The close-loop transfer function is:

$$
C(s I-A-B K)^{-1} B=\frac{0.1171}{s^{3}+0.2236 s^{2}+0.03337 s+0.00171}
$$

\section{Simulation Results}

The mathematical model of the system and the implementation for the control system will be described below. The orbit controller is then designed for the purpose of keeping the satellite in its nominal orbit.

Checking the system stability. The impulse response curve was plotted to investigate the system's stability. The angular velocity was $0.001 \mathrm{rad} / \mathrm{s}$, which was a 90 -min orbit in Figure 5 . This figure shows that the control input $u_{1}$ affected the output $y_{2}$, and that $u_{2}$ influenced both outputs $y_{1}$ and $y_{2}$. The impulse response kept growing which showed that the system was unstable.

Figure 6 shows the pole-zero map of the four-state variable model which established whether the system was stable or unstable. This figure shows that the system was unstable because the poles were situated on the right-hand side of the s-plane.

Figure 7 shows the impulse responses when the angular velocity was $7.3 \times 10^{-5}$. This corresponded to a 24-hour orbit (the geostationary orbit). As shown in the figure, the control input $u_{1}$ affected output $y_{2}$, and $u_{2}$ influenced both outputs $y_{1}$ and $y_{2}$. The impulse response kept 


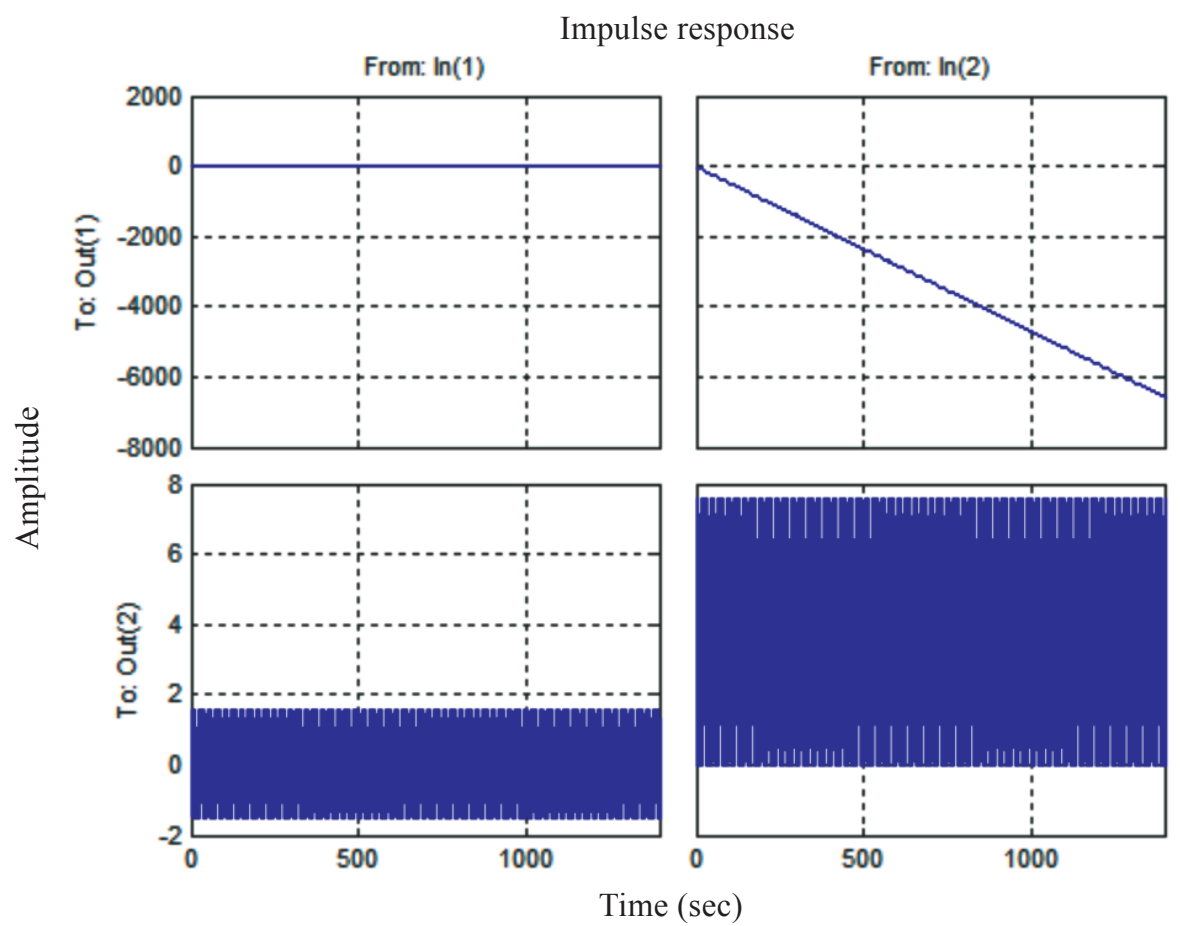

Figure 5. Impulse response of a four-state model in low earth orbit.

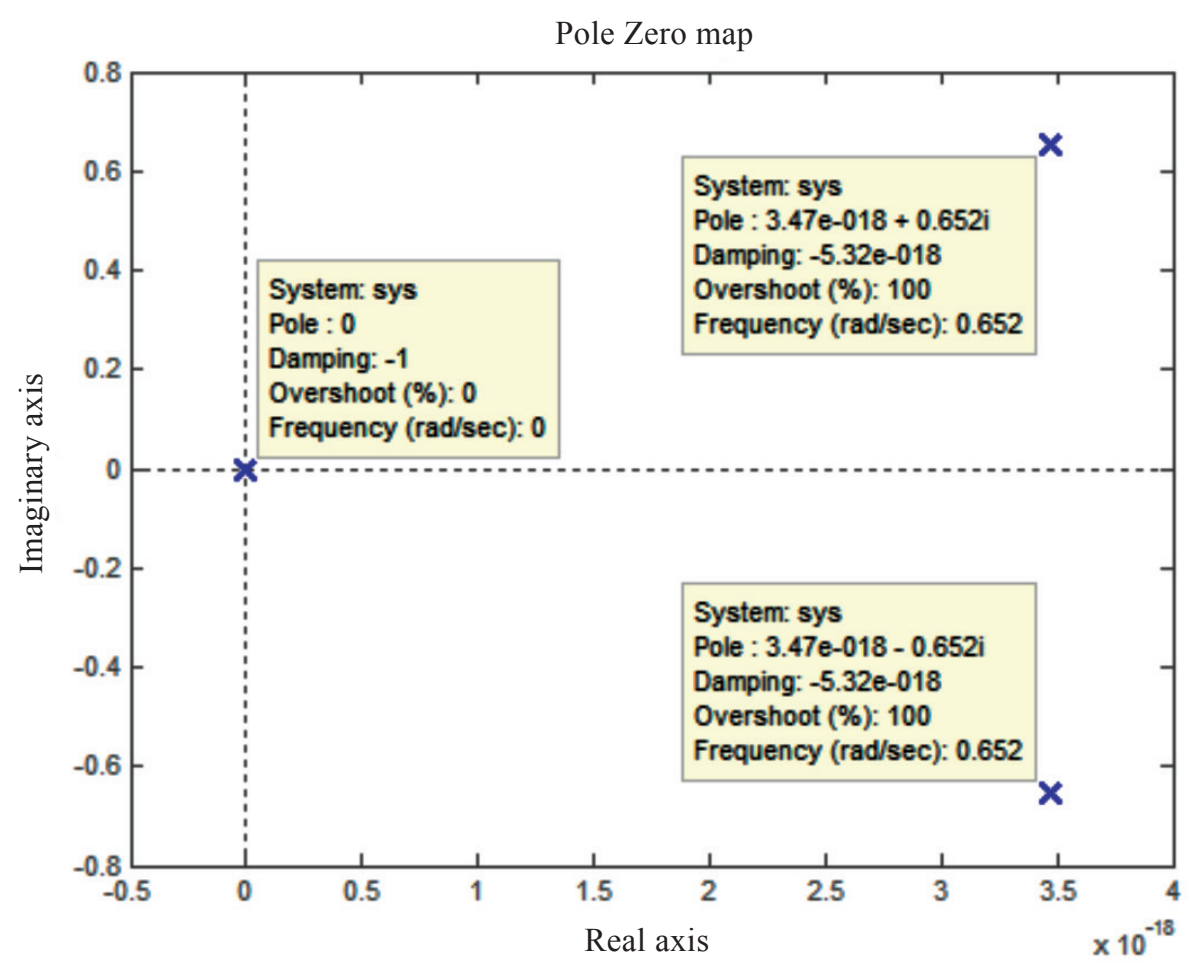

Figure 6. Pole-zero map of the four-state model in low earth orbit. 


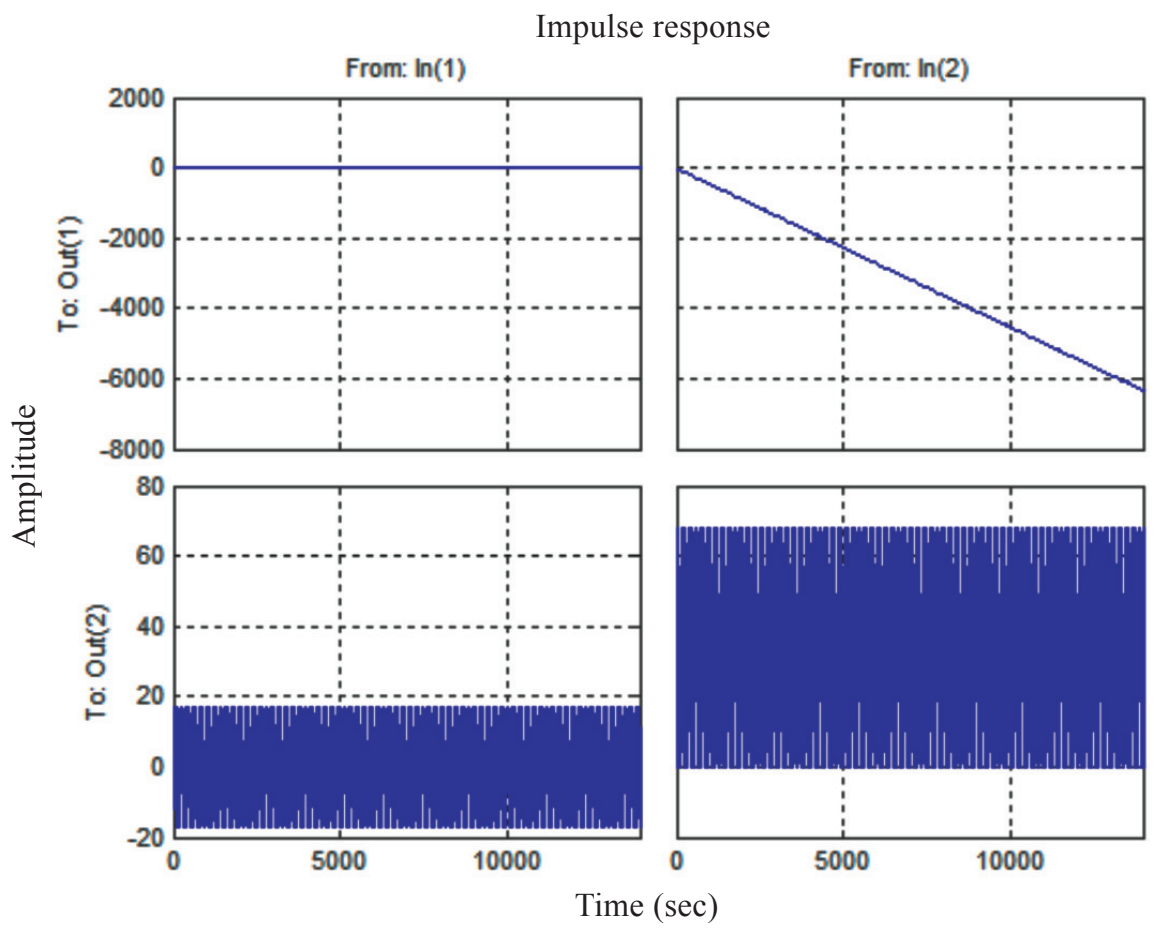

Figure 7. Impulse response of the four-state model in geostationary orbit.

growing, showing the system to be unstable. Figure 8 shows the pole-zero map of the fourstate variable model to establish whether the system was stable or unstable. As the system had repeated poles on an imaginary axis, it was marginally stable. Consequently, the system needed to be controlled so that the poles would be shifted to the left-hand side of the s-plane to ensure stability.

Checking the three-state model. Figure 9 illustrates the pole-zero map of the three-state variable model to establish whether the system was stable or unstable. With the poles located on an imaginary axis, the system was marginally stable. Therefore, the system needed to be controlled so that the poles would be shifted to the left-hand side of the s-plane to ensure stability. As shown in Figure 10, the impulse responded when the angular velocity was 7.3 $\times 10^{-5}$. For the three state variables, it may be seen in Figure 10 that the input influenced the output. This system was unstable according to the impulse response. Therefore, this system was considered a close-loop system with state variable feedback.

Pole placement configuration. The desired pole location of the system was established to determine whether the state feedback controller was stable or unstable. The desired poles were located at $-1,0.0748+\mathrm{j} 0.129$ and $0.0748-0.129$. The overshoot was $16.3 \%$ and the damping ratio was 0.5 as shown in Figure 11. A step response was illustrated for the third-order system with a close-loop transfer function as shown in Figure 12. For this system, the damping ratio was chosen at $\xi=0.5$, which corresponded to a $17 \%$ overshoot and a settling time of $2 \%$. This system was in a stable condition for about 80 seconds. The impulse response of the third-order system is as shown in Figure 13.

Initial condition of the three-state matrix. The response of the system to the given 
Pole Zero map

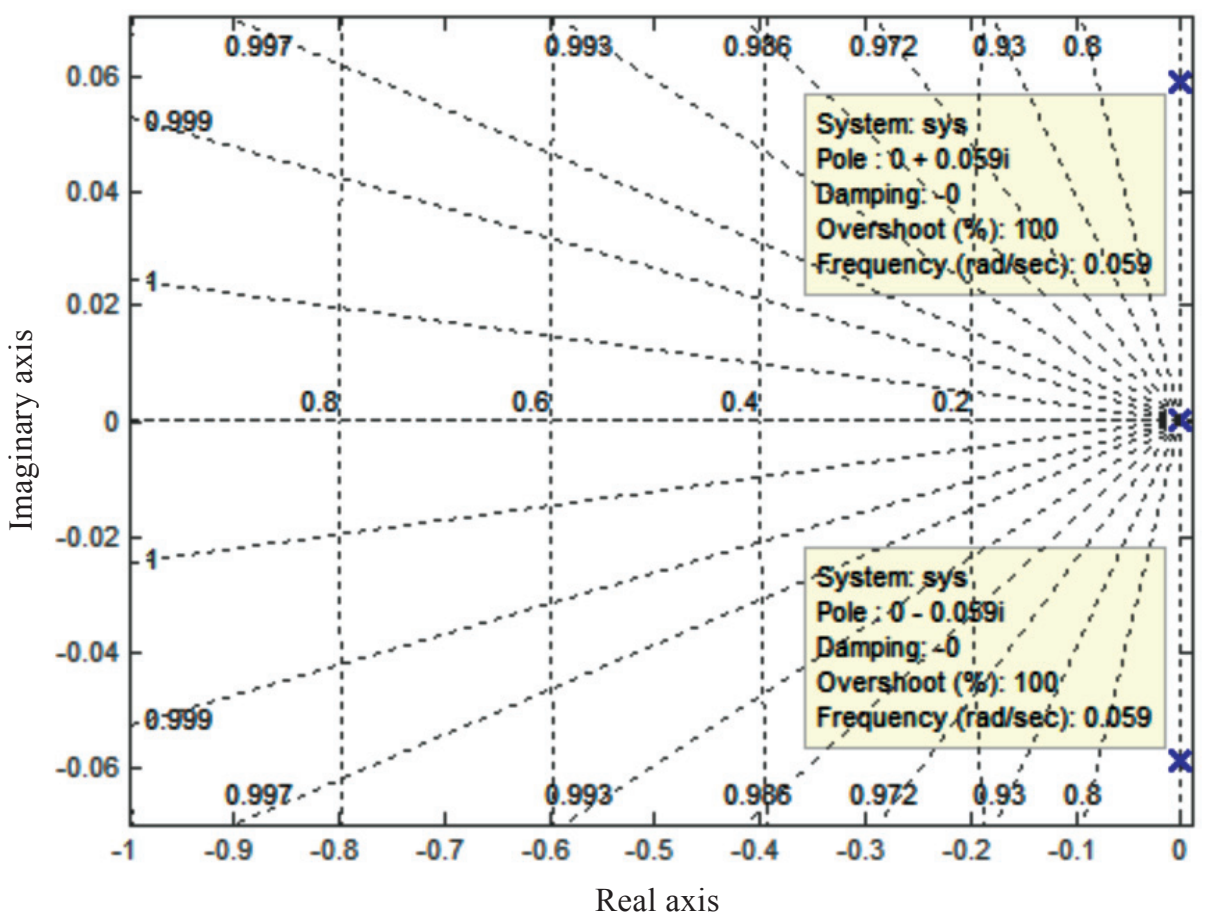

Figure 8. Pole-zero map of the four-state model in geostationary orbit.

Pole Zero map

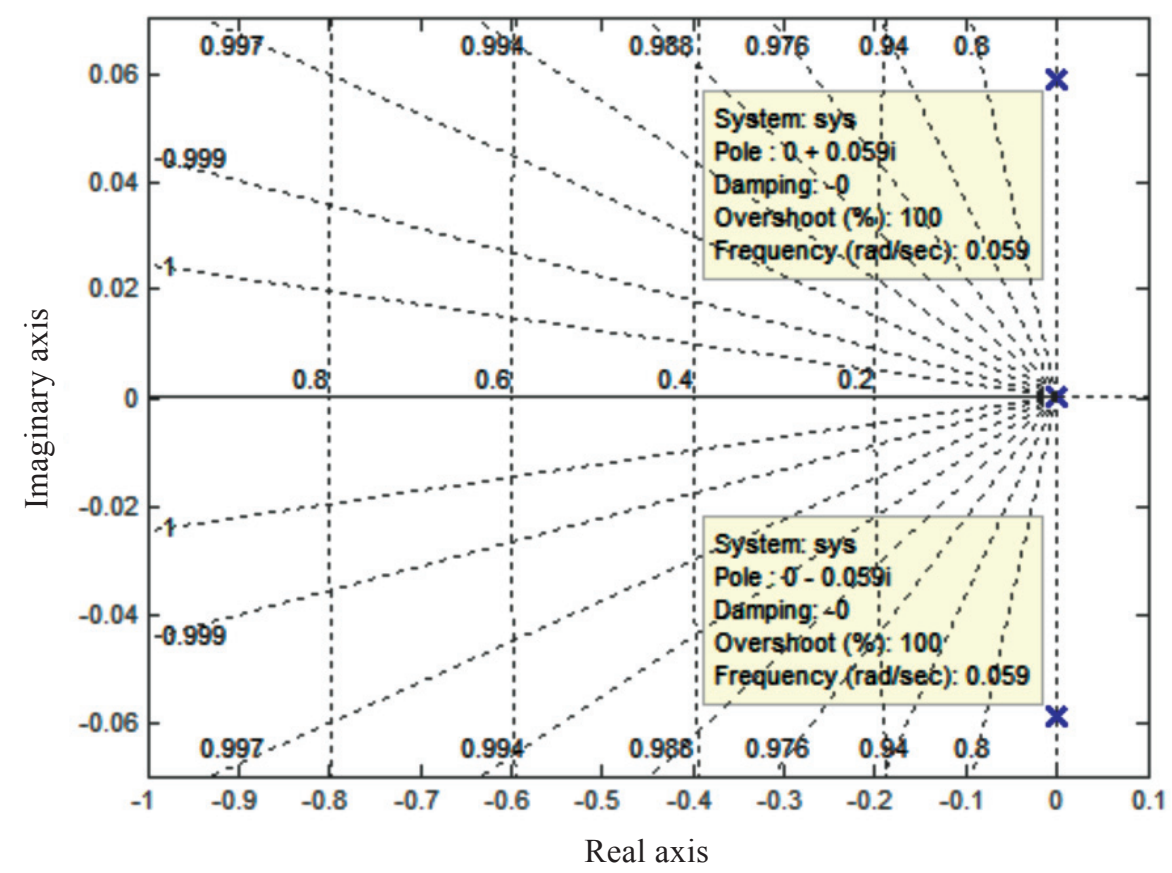

Figure 9. Impulse response of an open-loop system. 
Impulse response

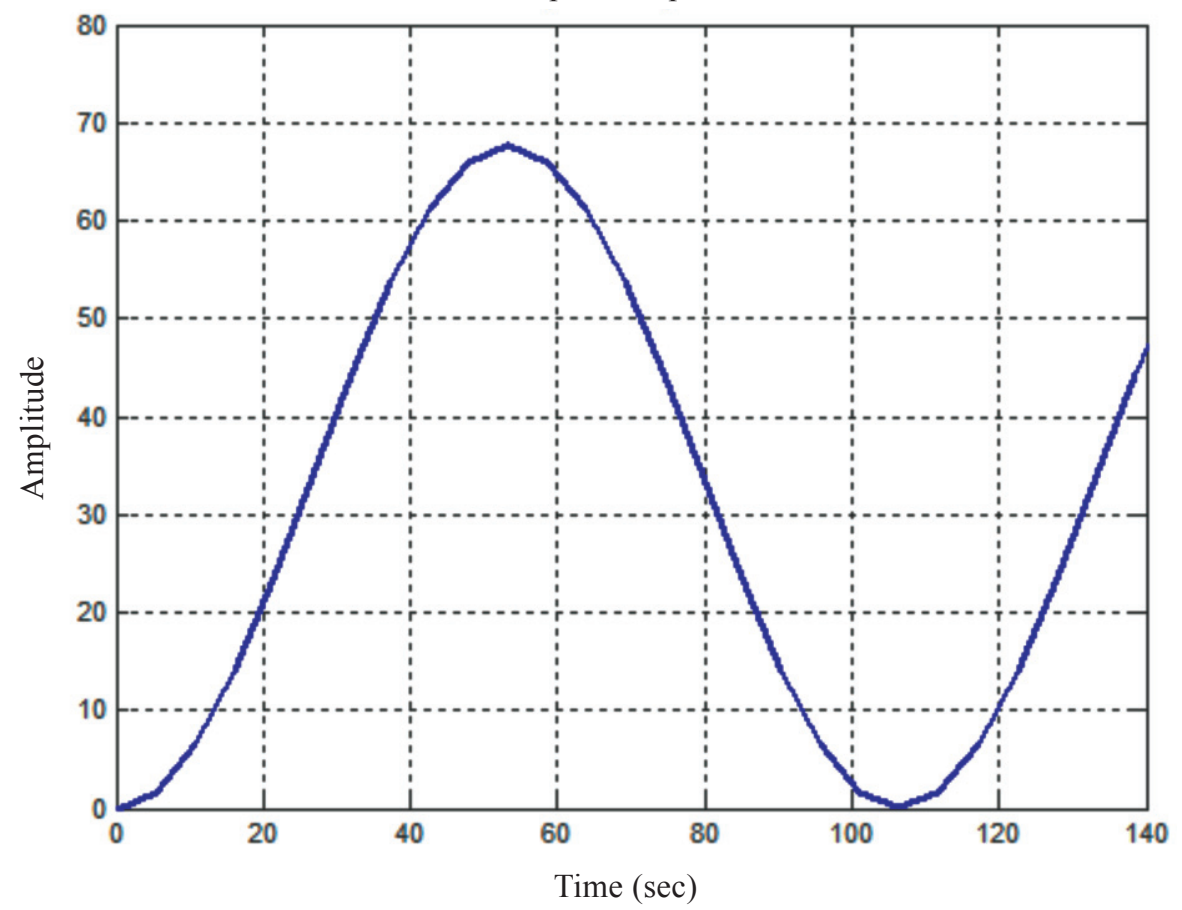

Figure 10. Step response of an open-loop system.

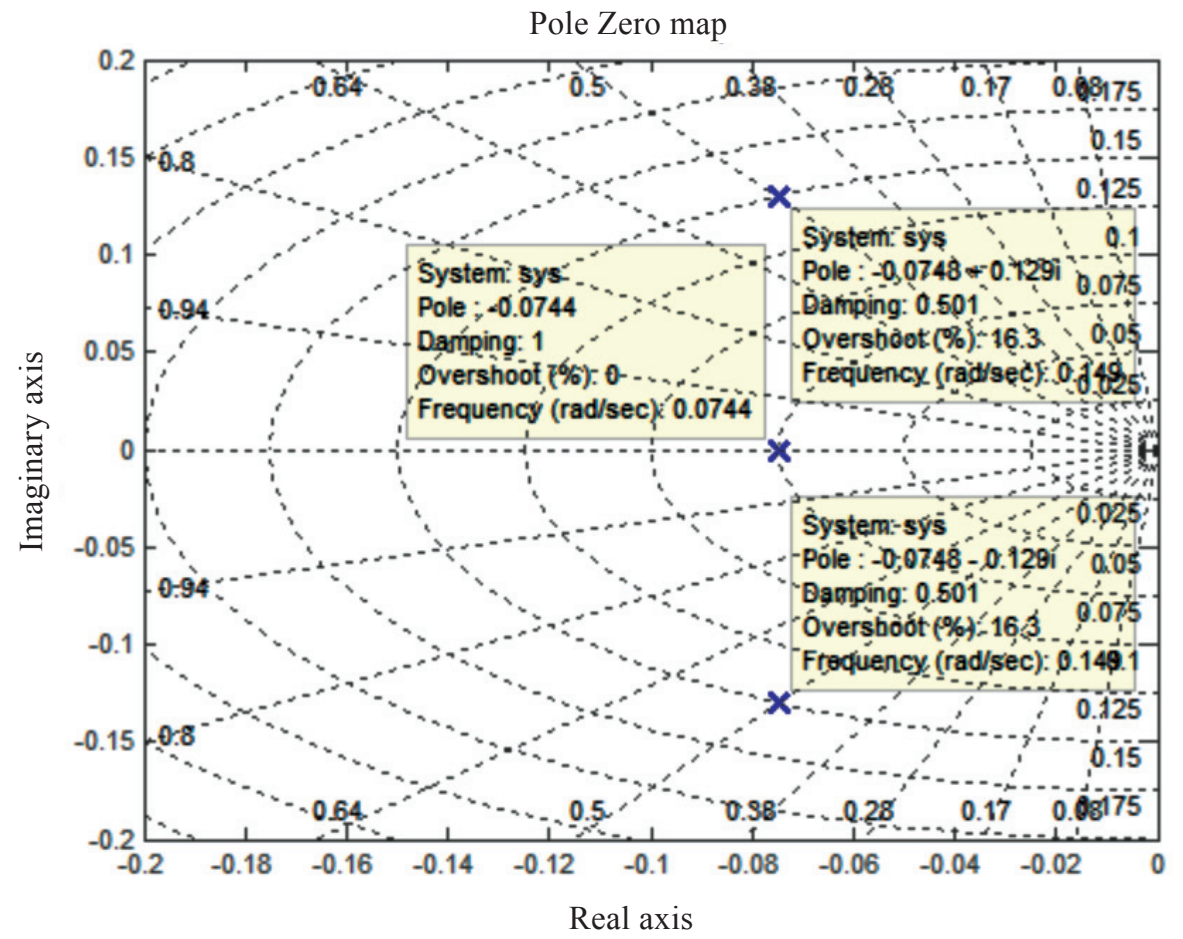

Figure 11. Pole placement configuration. 


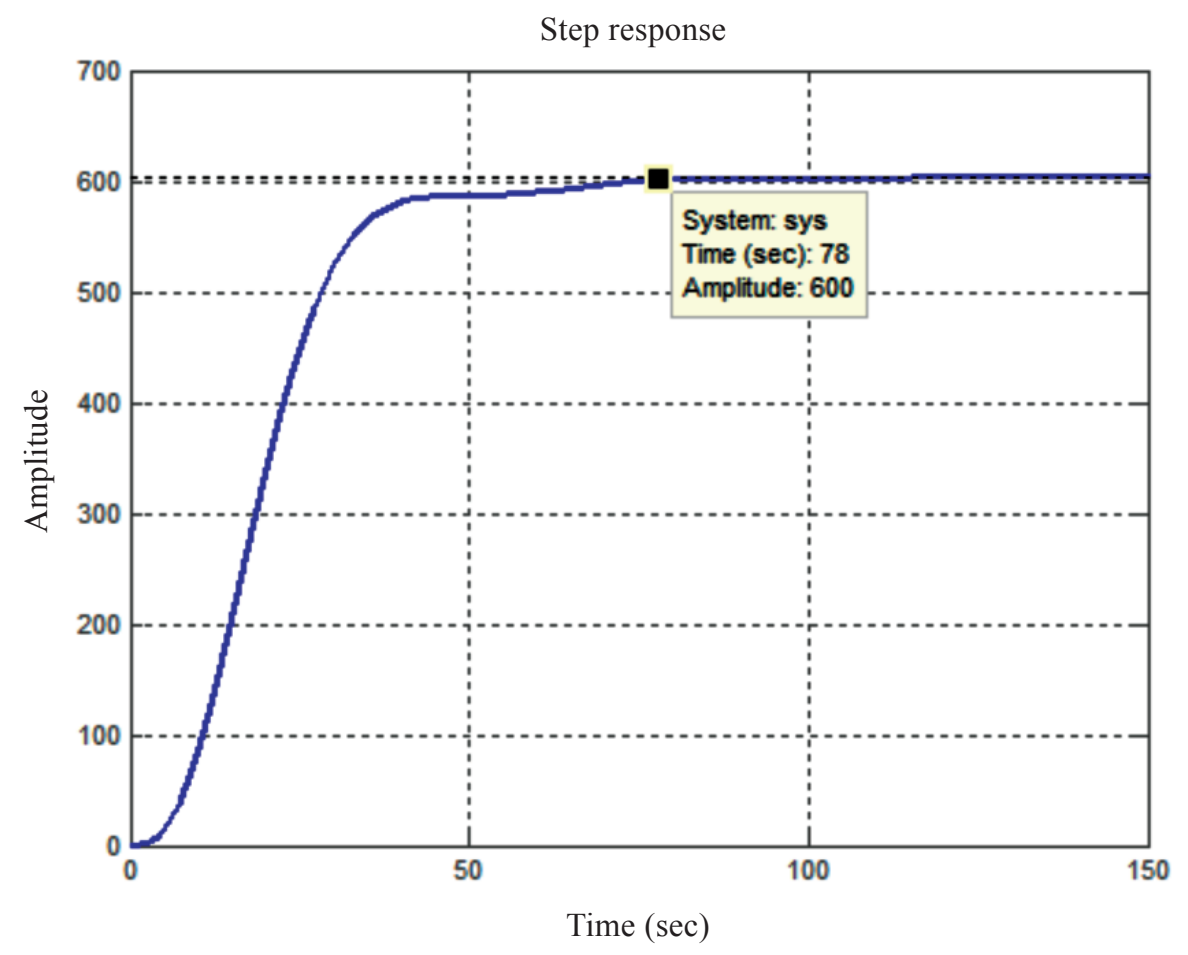

Figure 12. Step response of a state feedback controller.

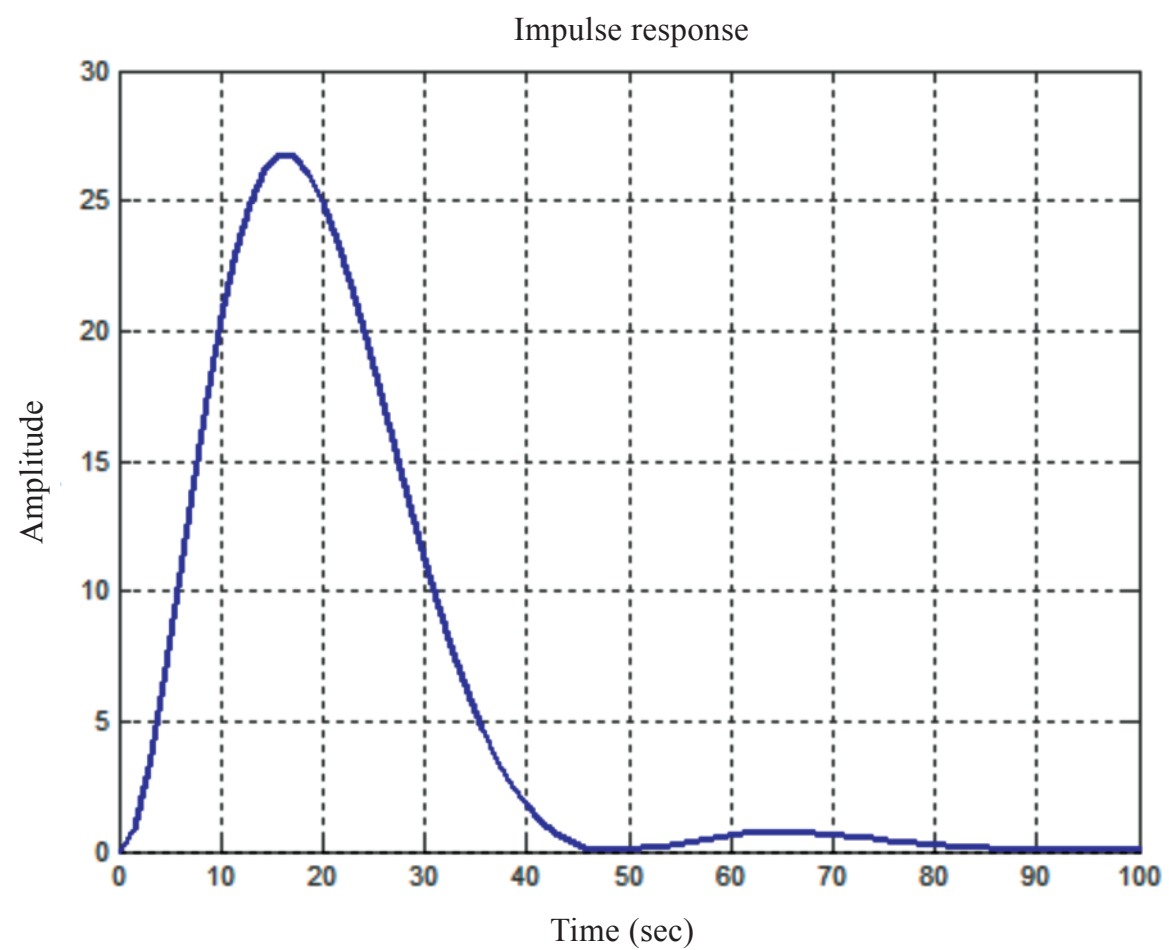

Figure 13. Impulse response of a state feedback controller. 
initial condition was determined. The resulting response curves are shown in Figure 14. The response curves were acceptable for the system.

Linear state feedback. Figure 15 shows the impulse response of a closed-loop system. The closed-loop system was formulated by using a state feedback control law with a gain matrix. The step response of the closed-loop system is shown in Figure 16. The settling time was 78.8 seconds. Figure 17 shows the linear simulation results of the closed-loop system. The time response of continuous linear systems to arbitrary inputs was simulated.

\section{CONCLUSION}

This work was initially done to study and analyze theoretically the orbit controller for a geostationary satellite. After generating the simulation for the supposed orbit controller, it was compared with the standard design for an orbit controller. The proposed orbit controller design could be linked to a communication satellite by comparing it with the simulation approaches of the existing system. Confirmation of the supposed design was ascertained and verified by running the simulation with the parameters of the orbit controller of standard design. To implement the orbit controller of the satellite, the stable state model is needed. It should be tried with more data and parameters before the controller is chosen. For any satellite to progress from a concept to an actual orbiting satellite, properties such as stability and control derivatives, which determine the characteristics of the satellite, must be determined. This research has attempted to design an orbit controller for a satellite orbiting in a circular orbit. The purpose of the research was to create a mathematical model for the circular orbit. These data of the selected orbit were based on the current satellite technology, which includes modelling analysis. The orbit controller was established by the state space equations. The assigned poles and close-loop eigenvalues stabilized the system. The controllability of the system via state feedback was in an adequate condition. In situations where the plant was not controllable, using the feedback controller
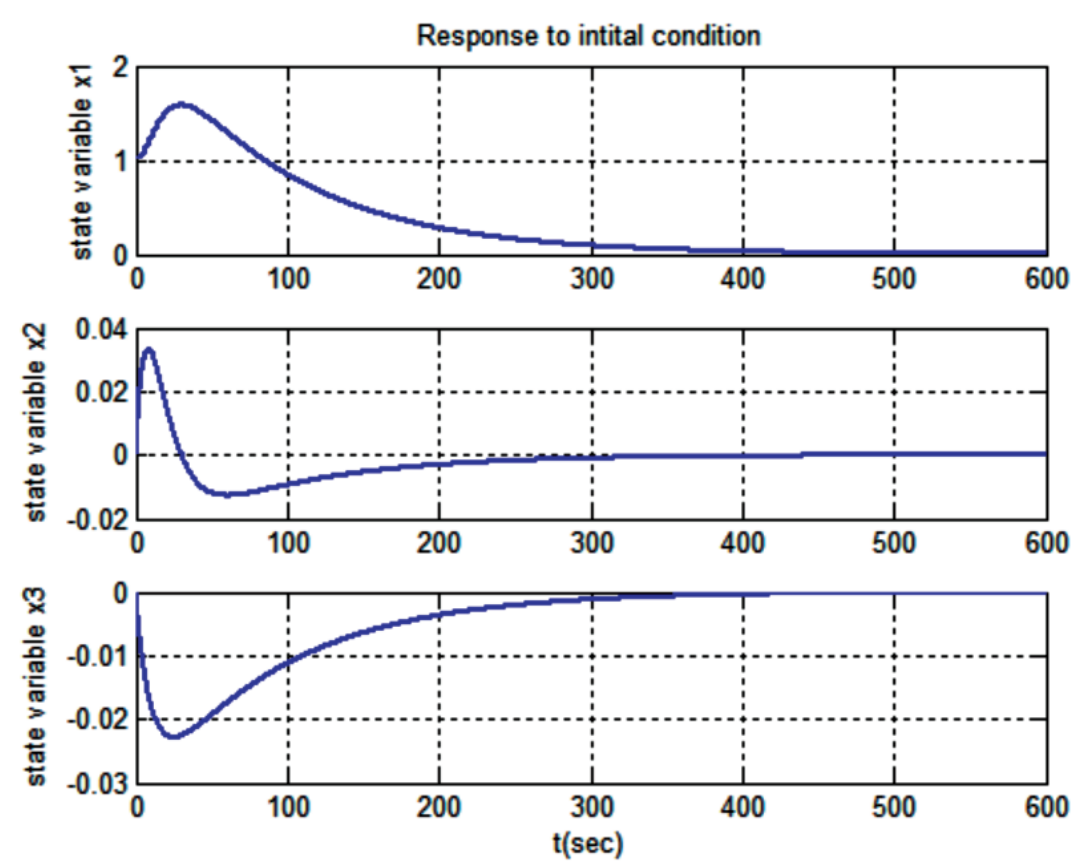

Figure 14. Response curve of the initial condition. 


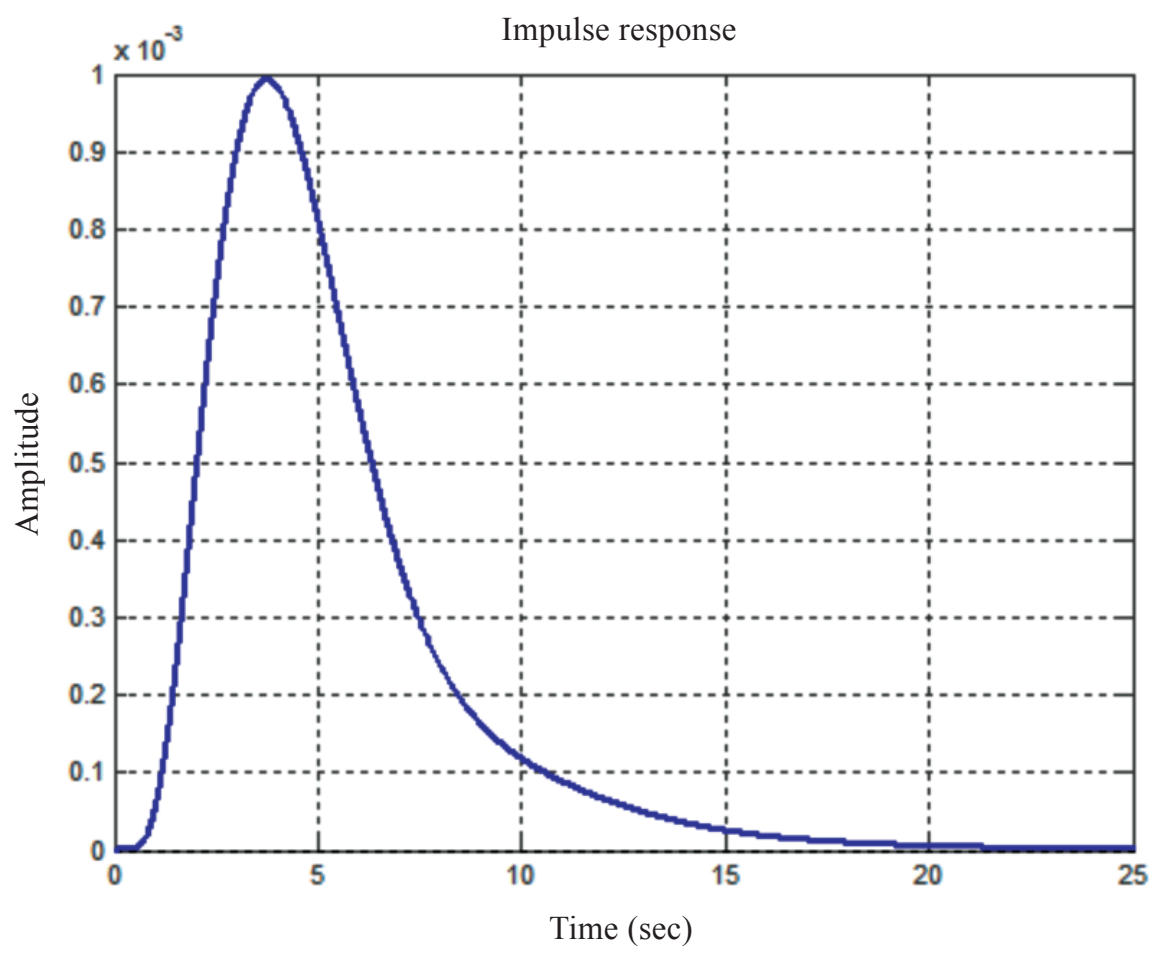

Figure 15. Impulse response of a closed-loop system.

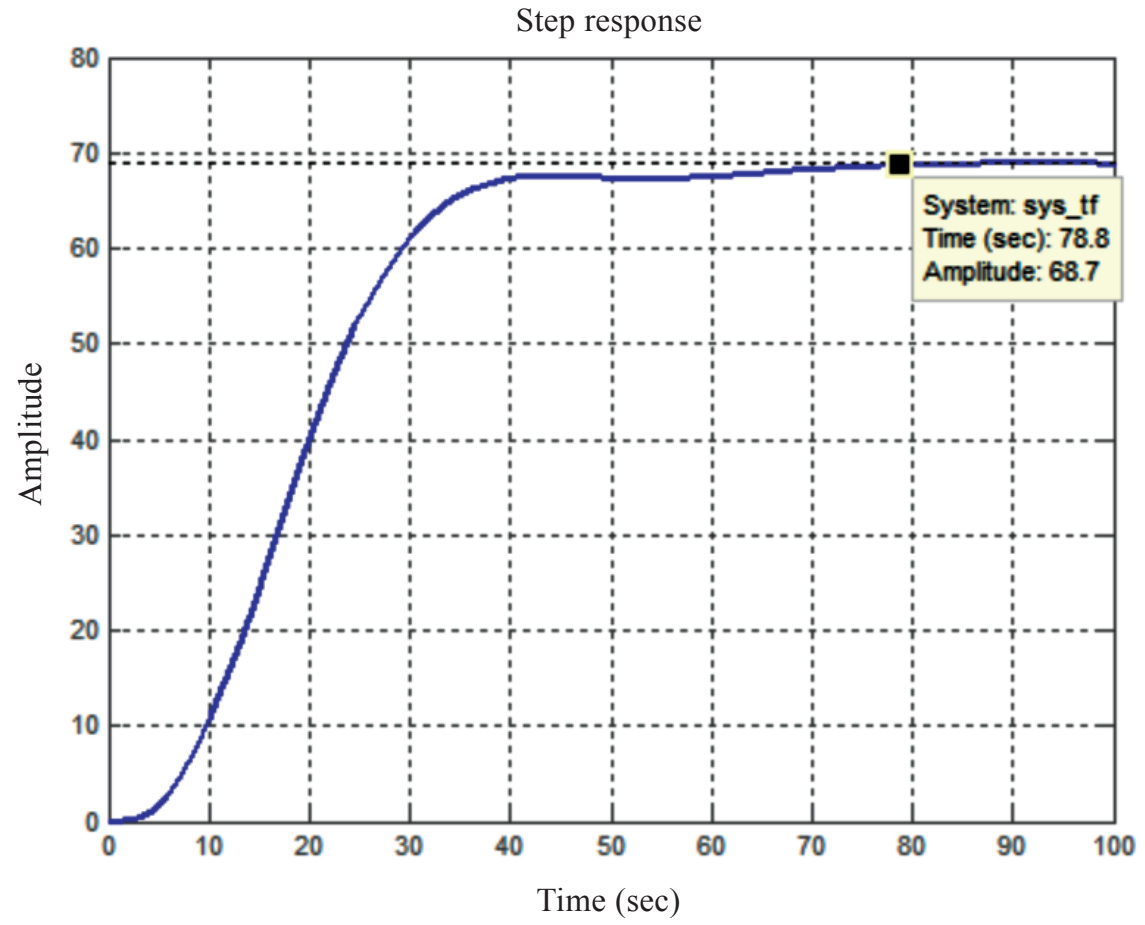

Figure 16. Step response of the closed-loop system. 


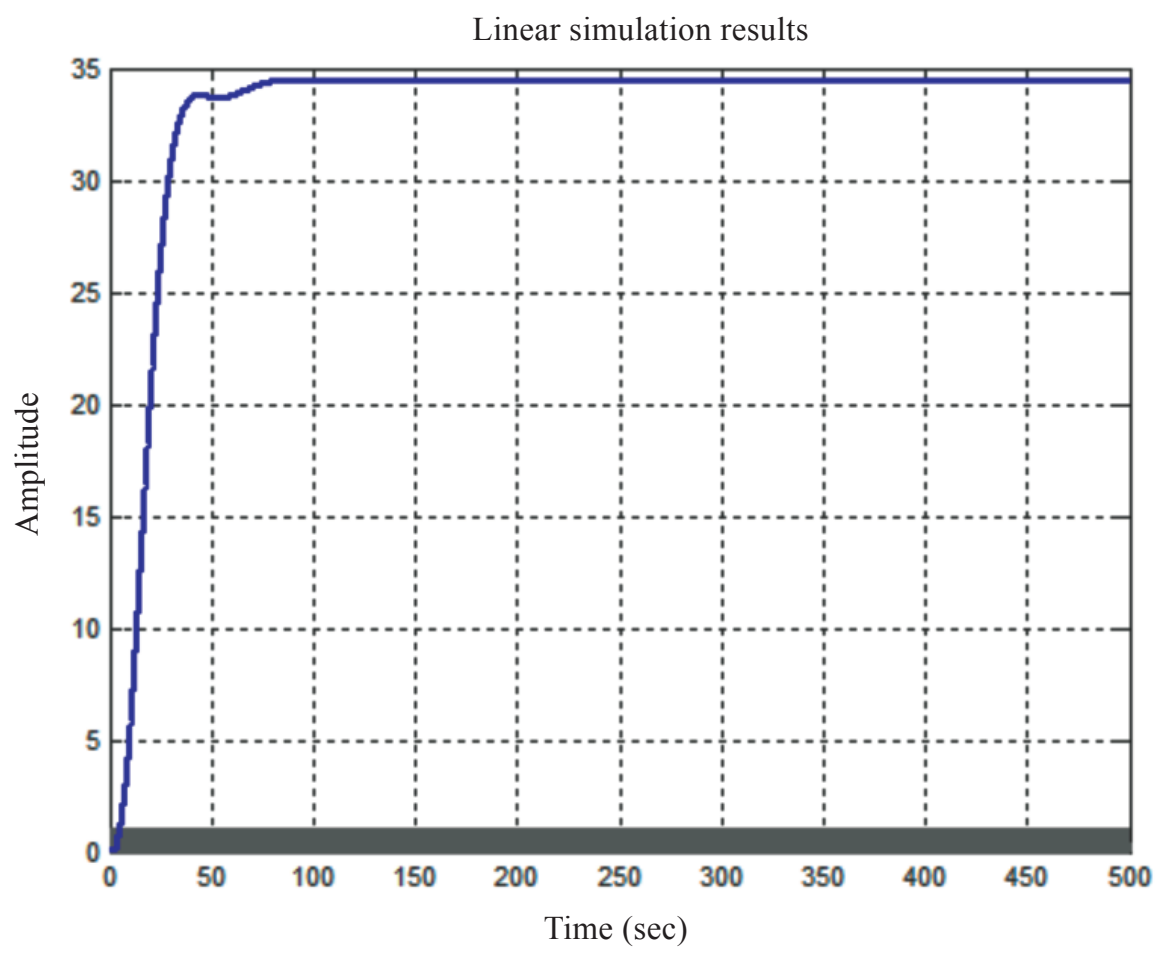

Figure 17. Linear simulation results of the closed-loop system.

can stabilize the unstable plant. In this system, the orbit controller design was considered for different possible controllable options. The response curves represented the stability of the system. The requirements of the orbit controller were designed by computing with MATLAB. It is very important to control the altitude of a satellite used for communications in a geostationary orbit. Hence this simulation program could be exploited for satellites orbiting around the earth at several altitudes to design the on-board propulsion system, and also to evaluate the amount of fuel necessary for the individual life of the satellite.

Date of submission: October 2011

Date of acceptance: June 2012

\section{REFERENCES}

Dorf, RC \& Bishop, RH 2011, Modern control systems, 12th edn, USA.

Gu, D-W, Petkov, PH \& Konstantinov, MM 2005, 'Robust control design with MATLAB', USA.

Maini, AK \& Agrawal, V 2011, 'Satellite technology: principles and applications', Wiley, Chichester, United Kingdom.

Malik, MA, Zaidi, GA, Aziz, I \& Khushnood, S 2001, 'Modeling and simulation of an orbit controller for a communication satellite', Pakistan.

Maral, G 2009, 'Satellite communications systems', United Kingdom.

Richharia, M \& Westbrook, LD 2011, 'Satellite systems for personal applications: concepts and technology', United Kingdom. 